\title{
reging
}

Revista Legado de Arquitectura y Diseño

ISSN: 2007-3615

ISSN: 2448-749X

legado_fad@yahoo.com.mx

Universidad Autónoma del Estado de México

México

\section{HABITABILIDAD Y RELACIONES SOCIO-ESPACIALES EN CONJUNTOS HABITACIONALES DE INTERÉS SOCIAL. CASOS DE ESTUDIO EN ENSENADA, BAJA CALIFORNIA, MÉXICO}

Organista-Camacho, Mariel; Tello-Peón, Lucía

HABITABILIDAD Y RELACIONES SOCIO-ESPACIALES EN CONJUNTOS HABITACIONALES DE INTERÉS

SOCIAL. CASOS DE ESTUDIO EN ENSENADA, BAJA CALIFORNIA, MEXICO

Revista Legado de Arquitectura y Diseño, vol. 2019, núm. 26, 2019

Universidad Autónoma del Estado de México, México

Disponible en: http://www.redalyc.org/articulo.oa?id=477961406009

Esta obra está bajo una Licencia Creative Commons Atribución-NoComercial-SinDerivar 4.0 Internacional. 


\title{
HABITABILIDAD Y RELACIONES SOCIO-ESPACIALES EN CONJUNTOS HABITACIONALES DE INTERÉS SOCIAL. CASOS DE ESTUDIO EN ENSENADA, BAJA CALIFORNIA, MÉXICO
}

\author{
HABITABILITY AND SOCIO-SPATIAL \\ RELATIONSHIPS IN SOCIAL HOUSING \\ COMPLEXES. STUDY CASES IN ENSENADA, BAJA \\ CALIFORNIA, MEXICO
}

\author{
Mariel Organista-Camacho mariel.organista@uabc.edu.mx \\ Universidad Autónoma de San Luis Potosí, México \\ Lucía Tello-Peón xlucha.tello@gmail.com \\ Universidad Autónoma de Yucatán, México
}

Revista Legado de Arquitectura y Diseño, vol. 2019, núm. 26, 2019

Universidad Autónoma del Estado de México, México

Recepción: 03 Marzo 2019 Aprobación: 31 Mayo 2019

Redalyc: http://www.redalyc.org/ articulo.oa?id=477961406009
Resumen: El estudio de la habitabilidad debe considerar requerimientos existenciales, proxémicos y contextuales en donde se propicie el bienestar humano a partir de las relaciones socio-espaciales. El objetivo del presente artículo es mostrar los hallazgos de la estimación de habitabilidad en conjuntos habitacionales de interés social en la ciudad de Ensenada, Baja California. Se considera un enfoque metodológico mixto. Se desarrolla y aplica cuatro instrumentos de análisis: cuestionario de habitabilidad, test de técnicas proyectivas, bases de datos y fotografías terrestres y aéreas. El modelo teórico de habitabilidad se basa en siete dimensiones: subsistencia, protección, afecto, entendimiento, participación, identidad y libertad. Se seleccionaron cuatro conjuntos habitacionales con un total de 5443 viviendas de interés social. Se aplicaron al azar 266 cuestionarios. Los resultados mostraron un patrón de baja o nula participación. La fragmentación espacial se presenta como vía de protección ante la inseguridad. La horizontalidad del conjunto favorece a cinco dimensiones de la habitabilidad (subsistencia, protección, afecto, entendimiento e identidad) y mejora la percepción de las condiciones socio-espaciales hacia el interior del conjunto; mientras que en los conjuntos verticales fue mayor la relación con los espacios exteriores y la ciudad. Se concluye que la habitabilidad depende de un complejo entramado de mediaciones entre lo privado, lo común y lo público. El entorno condiciona las relaciones sociales, las cuales se consideran impersonales, apresuradas y de conveniencia. En lo espacial, se presentan espacios dispersos, desorganizados y fragmentados que funcionan como medio de transición, lo que afecta negativamente a la habitabilidad.

Palabras clave: conjunto habitacional, habitabilidad, relación socio-espacial, vivienda social.

Abstract: The study of habitability must consider existential, proxemics and contextual requirements where human well-being is fostered from socio-spatial relationships. The aim of this article is to show the findings of the estimation of habitability in four social housing complexes in Ensenada, Baja California. A mixed methodological approach was applied. Four instruments were developed and applied: habitability questionnaire, test of projective techniques, databases and terrestrial and aerial photographs. The theoretical model of habitability is based on seven dimensions: subsistence, protection, affection, understanding, participation, identity and freedom. Four housing complexes were intentionally selected with a total of 5443 social housing units. 266 questionnaires were applied. The results 
showed a pattern of low or null participation. Spatial fragmentation occurs as a way of protection against insecurity. The horizontality of the complex supports five dimensions of habitability (subsistence, protection, affection, understanding and identity) and improves the perception of socio-spatial conditions towards the interior of the housing complex; while in vertical housing complex the relationship with the outer spaces and the city was greater. It is concluded that habitability depends on a complex network of mediations between the private, the common and the public. The context affects social relationships which are considered impersonal, hurried, also considered convenience relationship. In the spatial aspects, housing complexes are scattered, disorganized and fragmented spaces. Those aspects affect habitability.

Keywords: housing complex, habitability, socio-spatial relationship, social housing.

\section{INTRODUCCIÓN}

La constitución del ser en el mundo parte del sistema de relaciones que se establecen con los otros y con el espacio. Su habitar deriva a uno de los fenómenos más puros de la Arquitectura: la habitabilidad, la cual se relaciona intrínsecamente con requerimientos proxémicos, contextuales y existenciales del individuo, por lo que su estudio debe abordar la esfera social y espacial.

Castells (2004) considera al espacio como una estructuración de elementos ligados a lo social, que conforman una relación indisoluble entre el ser humano y su medio en una búsqueda por subsistir. Así, el espacio moldeado o modificado adquiere una función y un significado social.

Una concepción arquitectónica errónea

del conjunto habitacional puede generar

VIVIENDAS INCONEXAS ENTRE SÍ,

VIALIDADES PARA EL TRAFICO Y ESPACIOS EXTERIORES ABANDONADOS EIMPERSONALES.

La escala de ciudad, según Saunders (2011: 13-51), Gottdiener (1985: 70-75) y Jean Rémy \& Liliane Voyé (1974: 193-226), constituye el espacio en el que se instaura la moderna sociedad industrial, donde el sujeto que la habita se expone a múltiples interdependencias materiales, cuyas necesidades humanas se relacionan mayormente con fuerzas económicas. De particular interés es abordar los conjuntos habitacionales de interés social. Y precisamente, de acuerdo con Gehl (2009), son las características espaciales las que condicionan el sobrevivir, la conducta humana y las valoraciones de la habitabilidad. Por lo que, una concepción arquitectónica errónea del conjunto habitacional puede generar viviendas inconexas entre sí, vialidades para el tráfico y espacios exteriores abandonados e impersonales.

En México, esta connotación de vivienda de interés social se asocia con aquellos inmuebles destinados principalmente para los trabajadores, con énfasis en facilitar la adquisición de vivienda a quienes tienen menores ingresos (Sánchez-Corral, 2012). Para ello, el Gobierno de México oferta, a través del Programa de Vivienda Social 2019, apoyos para que la población de bajos ingresos con necesidad de vivienda y sin acceso a recursos o financiamiento suficiente puedan obtener una vivienda 
adecuada. La gestión de los apoyos se da en dos vertientes: producción social de vivienda y esquemas de cofinanciamiento, que básicamente consisten en un crédito hipotecario por medio de o INFONAVIT , FOVISSSTE entre otras entidades ejecutoras, que se complementa con subsidio y ahorro de la persona beneficiaria.

Asimismo, el hecho de compartir espacios y servicios comunes referido por Monnet (1996: 11-25) como "el arte de vivir juntos mediado por el espacio" tiene que ver con la socialización en la ciudad. Los conjuntos habitacionales de dicha índole son, en ocasiones, concebidos como una ciudad dentro de otra y llegan a ser vistos como espacios colectivos separados del resto de la ciudad. En gran medida, un factor que genera esta segregación son los problemas sociales típicos de la sociedad actual, como inseguridad, robo, vandalismo, drogadicción, entre otros. Los habitantes se ven obligados a establecer barreras físicas o simbólicas para su protección frente al caos que supone el exterior. Así, las relaciones sociales pasan a un segundo plano. En esa búsqueda de protección y seguridad, se renuncia al espacio comunitario exterior y se opta por la privatización del mismo.

El ser humano evidencia una necesidad de refugio donde la habitabilidad alcanza los niveles de subsistencia y protección a costa de una organización espacial que sólo permite el contacto controlado con aquellos que le resultan familiares al individuo. Sin embargo, el hecho de aislarse del mundo exterior y de situar como prioridad la protección frente al establecimiento de relaciones sociales deviene afectaciones de otras necesidades básicas de la habitabilidad como: afecto, participación, identidad y libertad.

Con relación al desarrollo de vivienda de interés social en México, se le ha dado prioridad en abatir el rezago habitacional más que a tratar de contribuir en la calidad espacial, por lo que, el nivel de habitabilidad podría verse limitado a satisfacer únicamente necesidades relacionadas con requerimientos existenciales y requisitos mínimos en lo legal y técnico.

Si bien, los estudios de la habitabilidad han sido abordados desde diversos enfoques; las categorías en las dimensiones, subdimensiones, variables e indicadores que intervienen en este fenómeno varían de acuerdo al problema de investigación planteado, a los casos de estudio seleccionados y al contexto específico de la zona analizada. Otros estudios en torno a la vivienda profundizan en variables aisladas por lo que estas investigaciones no pueden extrapolarse a una evaluación global de la habitabilidad en toda su complejidad.

El estudio de la habitabilidad como una totalidad que integre las relaciones espaciales del conjunto habitacional con el entorno inmediato y la ciudad será una vía para explicar la complejidad del espacio construido en contextos específicos y coadyuvará en el nivel de satisfacción de necesidades humanas, en la mejora de sus espacios y su contexto.

Con base en lo anterior, en este documento se estima la habitabilidad en cuatro conjuntos habitacionales de interés social ubicados en la ciudad de Ensenada, Baja California, a $110 \mathrm{~km}$ al sur de la frontera oeste 
México-Estados Unidos, a partir de una propuesta teórica articulada en siete dimensiones relacionadas con las necesidades básicas que engloban aspectos del ser y de los espacios. Las dimensiones refieren a la subsistencia, la protección, el afecto, el entendimiento, la participación, la identidad y la libertad y son abordadas desde el nivel de satisfacción que se logra por las relaciones del ser desde lo individual y colectivo en función de su interacción con el entorno. Estas siete dimensiones se desglosan con referencia en un marco teórico en la figura 1 . La importancia de identificar los principales factores de afectación de la habitabilidad en conjuntos habitacionales, y su relación socio-espacial será fundamental en la búsqueda de mejores alternativas de vivienda de interés social.

\section{METODOLOGÍA}

La propuesta de investigación involucrada en este estudio, se aborda desde un enfoque metodológico mixto, que recupera información cuantitativa para la estimación de la habitabilidad y sus relaciones espaciales y se complementa con información de corte cualitativo mediante observaciones directas en campo. La idea es obtener una representación de las condiciones del espacio habitable desde una perspectiva integral.

\section{Contexto del estudio}

El trabajo de campo se realizó durante el 2018, en cuatro conjuntos habitacionales de interés social de la ciudad de Ensenada B.C. Los conjuntos habitaciones y viviendas fueron seleccionadas con base en: i) Año de construcción, con un periodo de consolidación del conjunto mayor o igual a diez años; ii) Productor representativo de la región (se buscó que la edificación fuera hecha por algún organismo o compañía que haya realizado acciones de vivienda a nivel estatal desde su creación; se espera que esta cobertura estatal sea un reflejo de la vivienda mayormente ofertada en la zona); iii) Localización del conjunto fuera de la mancha urbana al momento de su edificación ; iv) Características físicas, como proporción vertical/horizontal, materiales, dimensiones de construcción y cantidad de viviendas; v) Reflejo de la oferta pública y privada de vivienda de la cual surgieron; es decir, que dicho conjunto habitacional seleccionado fuera una muestra de las políticas de vivienda que se han puesto en marcha y representativo de la oferta de vivienda de interés socialy; vi) Precio de la vivienda.

\section{Selección de las viviendas/habitantes}

Para obtener el tamaño de muestra se utilizó la herramienta de cálculos proporcionada por el Departamento de Sistemas Informáticos Integrales de la Facultad de Medicina en la Universidad Nacional del Nordeste (2017). De esta manera se insertó el total de viviendas de los cuatro conjuntos habitacionales, que conformaron la población de interés $(\mathrm{N})$ 
de 5 443. Se consideró un nivel de confianza de $90 \%$, con un valor de proporción $(\mathrm{P}=50 \%)$ y un error estadístico de $10 \%$. Con estos valores, el tamaño de muestra calculado fue de 246 , que corresponden a $4.5 \%$ del total. La selección de las 246 viviendas de interés, se realizó al azar manteniendo la cuota de proporcionalidad respectiva con base en la cantidad de viviendas de cada conjunto habitacional.

\section{Instrumentos}

Se utilizaron cuatro para la recuperación de información: i) Cuestionario de dimensiones de habitabilidad, ii) Test de técnicas proyectivas, iii) Fotografías (Cámara/Dron) e iv) información documental. Con el apoyo de dichos instrumentos, se analizó la influencia de las continuidades espaciales del conjunto habitacional con su entorno inmediato y mediato en función del nivel de satisfacción de las necesidades humanas. A continuación, una breve descripción de los instrumentos.

\section{Cuestionario de estimación de habitabilidad}

Tiene el propósito de recuperar información de las dimensiones y variables de mayor relevancia para la estimación de la habilidad y sus relaciones espaciales. La habitabilidad, como un rasgo latente asociado a la vivienda y a quien la habita, requiere de una métrica especial. Se parte de una división de siete dimensiones para conformar una estructura para su caracterización. El sustento teórico puntualiza a autores como: Neef (1986 citado en Salazar, 2012), Concello de Lugo y Agencia d'Ecologia (2010), García-Gómez (2013), Giglia (2001), Gómez Amador y Gómez Azpeitia (2010), Jirón y Toro (2004), Kirschenmann (1985), Landázuri y Mercado (2007), Maycotte Pansza (2007), Rueda (2003), Salazar (2012), Salgado, Aguillón y Carranza (2014), entre otros. Así, la estructura resultante se muestra en la figura 1 .

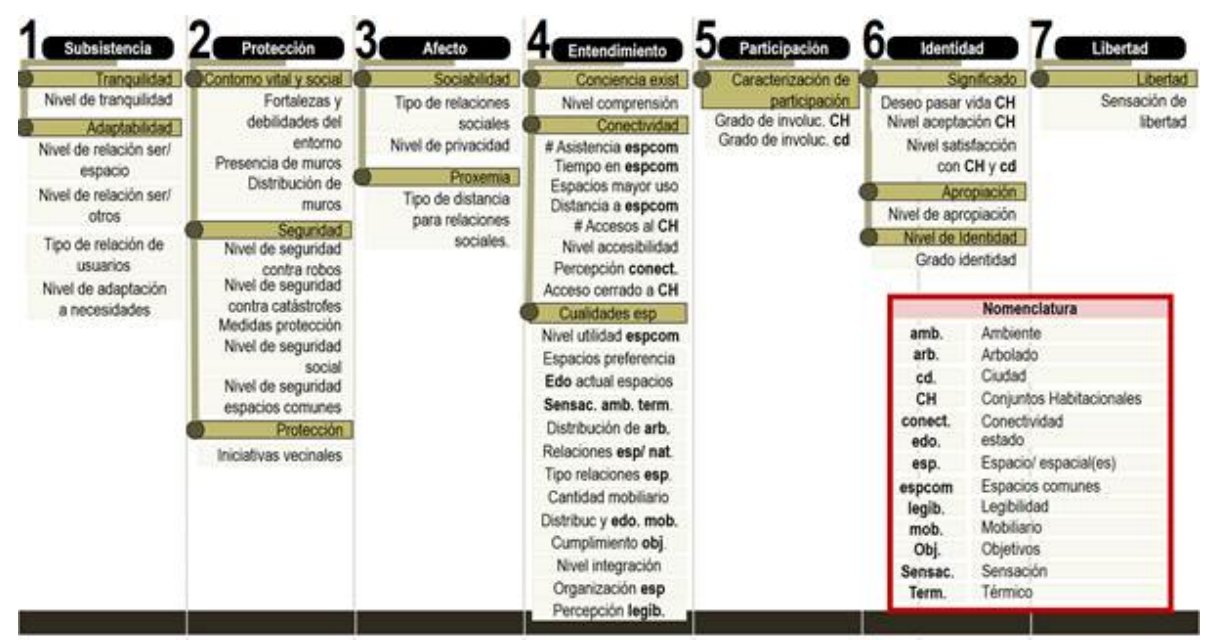

Figura 1. Dimensiones de la habitabilidad.

Fuente: Elaboración propia con base en los autores mencionados (2018). 
Esta estructura fue el punto de partida para la elaboración del cuestionario, que consistió de 45 enunciados. Cada enunciado fue revisado por cinco expertos, quienes evaluaron el nivel univocidad, con la intención que cada reactivo tuviera una y sólo una interpretación (Carrera \& Balsells, 2011: 1-25). En cuanto a la métrica de los indicadores, se utilizó un nivel de medición nominal dicotómico y polinómico para 10 enunciados orientados a registrar características meramente clasificatorias o presencia/ausencia de algún rasgo distintivo de la vivienda, por ejemplo, la presencia de muros, o el estatus de la propiedad, por mencionar un par de enunciados. Para las siete categorías del constructo habitabilidad, se utilizaron 26 indicadores en el nivel de medición ordinal basado en calidad (pésimo-excelente) y cantidad (nada-completamente). El resto de los enunciados del cuestionario utilizó la escala de razón para recuperar de forma precisa, algún indicador o característica de la vivienda, del entorno inmediato o de la ciudad. Para estimar la fiabilidad de las puntuaciones, se calculó el coeficiente Alpha de Cronbach. El valor obtenido fue 0.88 , lo que aporta evidencia de una adecuada fiabilidad de la información recuperada.

\section{Test de Técnicas proyectivas}

Tiene la finalidad de que el entrevistado pueda interpretar y explicar a preguntas directas relacionadas con: las razones de ciertos comportamientos, o lo que la acción de comprar, poseer o usar un producto o servicio significa para ellos. El test elaborado se articuló mediante las siguientes categorías de técnicas proyectivas: i) asociación de palabras, ii) pruebas de frases incompletas e; iii) interpretación de dibujos. Así, el instrumento posee 20 palabras de asociación, nueve frases incompletas y cuatro imágenes para su interpretación . La información recuperada permitió profundizar los resultados con relación a las dimensiones consideradas en el estudio.

\section{Fotografias}

Se tomaron series fotográficas en cada uno de los conjuntos habitacionales. Dichas fotografías permitieron analizar el encuadre: plano general; medición de la luz con fotos en diferentes horarios. Se recurre a fotografías aéreas con el uso de un dron e impresiones de pantalla con fotografías satelitales desde Google Maps (2018). El propósito es contar con información visual del espacio exterior e interior de cada conjunto de estudio.

\section{Información documental}

Se solicitaron planos de los modelos prototipo básico de los conjuntos habitacionales. Fue necesaria la revisión de planos de desarrollo urbano de la ciudad. La información se complementó con información relacionada 
a los conjuntos habitacionales proveniente de periódicos y bases de datos como las de INEGI.

\section{Intervención en campo}

La estrategia de intervención implementada para obtener la información de los dos instrumentos relacionados con la percepción del habitante (Cuestionario de Dimensiones de Habitabilidad y Test de Técnicas proyectivas) quedó delimitada en las cinco fases siguientes: a) Búsqueda y selección de aplicadores, b) Capacitación de aplicadores y preparación de material, c) Trabajo de campo, d) Recepción de instrumentos aplicados y e) Cumplimiento de las metas.

La estrategia de intervención para la toma fotográfica fue mediante cámara digital en tierra, y el apoyo de un dronógrafo para las capturas aéreas -fotográficas y de videos- con énfasis en la estructura del conjunto habitacional, la distribución de las viviendas y las conexiones viales dentro del conjunto y con la ciudad.

\section{Análisis de la información}

La información obtenida mediante el cuestionario y test de técnicas proyectivas se digitalizó en un archivo del programa estadístico SPSS v. 22. Se obtuvieron descriptivos básicos de los indicadores asociados a cada dimensión. En el caso de la información cualitativa se identificaron las ideas principales y se definieron las categorías de interés y la frecuencia de ocurrencias para cada una de ellas. La información fotográfica permitió complementar los análisis y la interpretación de la información.

\section{RESULTADOS Y DISCUSIONES}

Los cuatro conjuntos habitacionales $(\mathrm{CH})$ del presente estudio, conforman un espacio habitable constituido por un total de 5443 viviendas (figura 2). Cada conjunto presenta particularidades en su diseño, funcionalidad y conexiones con la ciudad, por lo que la descripción siguiente, pretende facilitar la interpretación de los hallazgos 


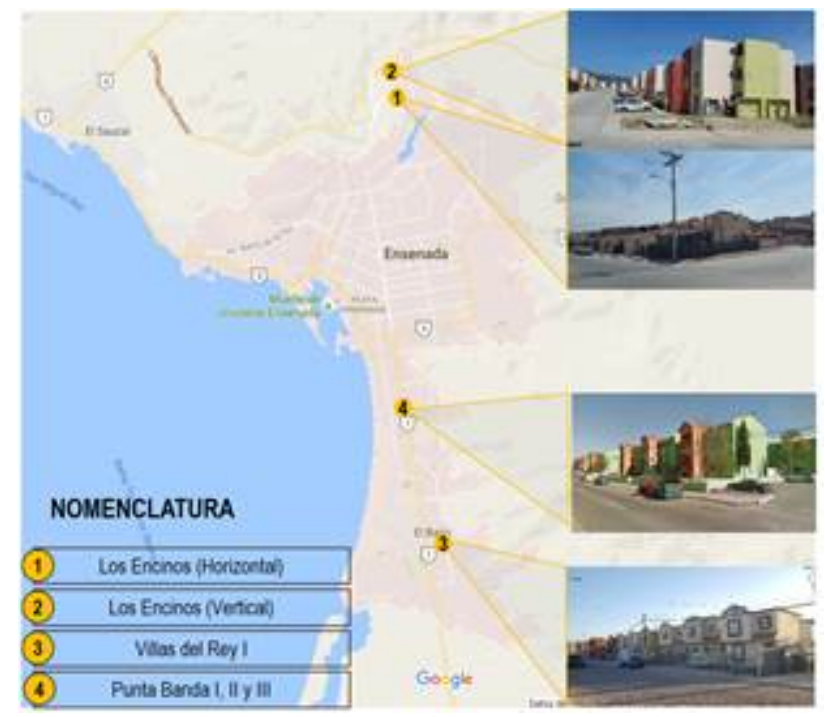

Figura 2. Cuatro conjuntos habitacionales de la ciudad de Ensenada, B.C. Fuente: Elaboración propia (2019).

CARACTERIZACIÓN DE LOS CONJUNTOS HABITACIONALES

\section{CH. Encinos horizontal}

Ubicado al norte de la ciudad, es un conjunto habitacional de interés social. Cuenta con 3684 viviendas de tipo horizontal. La información recuperada de este conjunto fue la proveniente de 107 viviendas, cantidad mayor al valor teórico estimado (66) mediante el algoritmo de tamaño de muestra. Se destaca que en $48 \%$ de las viviendas visitadas habitan cuatro o más. Las características de sus habitantes son: $38 \%$ cuenta con escolaridad preparatoria y $30 \%$ tiene estudios universitarios. $57 \%$ tiene menos de 5 años residiendo ahí. La tasa de empleo alcanza $71 \%$.

\section{CH. Encinos vertical}

Colinda con el CH de Encinos horizontal. Está conformado por 696 viviendas de tipo vertical. En este conjunto se recuperó información de 100 viviendas, cuota superior a la proporción teórica estimada de 61. En $56 \%$ de las viviendas habitan cuatro o más. Se destaca baja escolaridad de sus residentes, ya que $56 \%$ tiene primaria/secundaria y $29 \%$ preparatoria. La mayoría de sus residentes (71\%) tiene menos de 5 años de vivir en este conjunto. Solo $58 \%$ cuenta con empleo.

\section{CH. Villas del Rey I}

Ubicado al sur de la ciudad, es un $\mathrm{CH}$ de tipo horizontal. Cuenta con un total de 616 viviendas. Se recuperó la información de 93 de ellas, excediendo el valor teórico de tamaño de muestra de 61. En 59\% de las viviendas habitan cuatro o más. Se destaca por escolaridad alta, ya que 
$37 \%$ cuenta con estudios universitarios o posgrado. Es una comunidad con cierto arraigo, ya que 47\% tiene entre 11-20 años residiendo en el conjunto. $63 \%$ de sus residentes cuenta con empleo.

\section{CH. Punta Banda}

Ubicado al sur de la ciudad, cuenta con un total de 447 viviendas, es de tipo vertical. Es el más antiguo de los $\mathrm{CH}$. Se recuperó la información de 65 viviendas, cuota superior al valor teórico calculado (58). En 62\% de las viviendas habitan cuatro o más personas. La escolaridad que logra mayor porcentaje (42\%) fue preparatoria, mientras que $19 \%$ cuenta con estudios universitarios. Es un $\mathrm{CH}$ donde $51 \%$ tiene más de 20 años de residir ahí. $55 \%$ cuenta con empleo.

\section{Estimación de la habitabilidad}

La habitabilidad es una dimensión compleja que debe considerar las cualidades espaciales, estructura familiar, composición interna, el capital económico y social, el grado de desarrollo socioeconómico de la familia y la zona de residencia (Gazmuri, 2013: 32-47). La información previamente presentada, perfila al habitante típico de cada uno de los $\mathrm{CH}$ de estudio. La figura 3 muestra un polígono con las siete dimensiones de la habitabilidad y sus variables involucradas. Se utiliza una escala de 0-10 para facilitar su interpretación. En el polígono se muestran los valores relacionados con la habitabilidad de cada $\mathrm{CH}$.

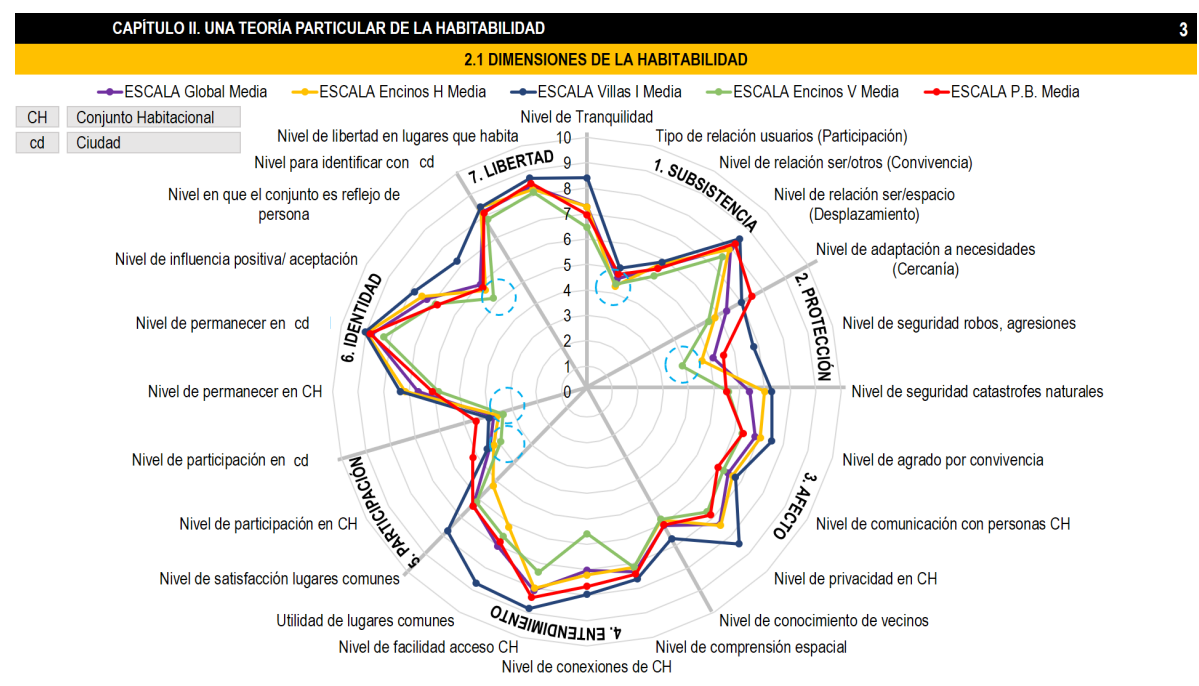

Figura 3. Dimensiones de habitabilidad.

Fuente: Elaboración propia (2018).

En lo que refiere a la subsistencia la variable desplazamiento presenta tendencia positiva al igual que el nivel de cercanía a lugares destinados a satisfacer necesidades básicas. El CH mejor evaluado fue Villas del Rey. El limitado interés por el colectivo mismo y el medio circundante se explica por las barreras físicas del conjunto que devienen barreras sociales. Se 
coincide con la idea de asociar la construcción de estos conjuntos como "viviendas sin ciudad" (Giglia, 2012).

Acerca de la protección, en tres de los cuatro $\mathrm{CH}$, la evaluación de esta dimensión mostró evaluación baja. La variable peor evaluada fue el nivel de seguridad contra robos o agresiones como producto de nichos sin salida y barreras físicas que impiden la vigilabilidad. Este grado de vigilabilidad se da principalmente para operaciones delictivas.

En la dimensión de afecto, los habitantes prefieren mantener cierto nivel de privacidad que buscar la convivencia o comunicación con otras personas del mismo conjunto. Si bien, Augé (1996) habla de la constitución del ser en el mundo a partir de un sistema de relaciones con otros o Lefebvre (2013) puntualiza que no hay espacio sin relaciones sociales, en estos casos, los espacios comunes pierden la importancia para el habitante, quien prefiere los espacios exteriores de tipo privado. La afluencia de personas es casi nula dado que la movilidad se rige por el automóvil.

En la dimensión de entendimiento, las plantas arquitectónicas lineales apoyaron la legibilidad urbana. Por su parte, Parrish (2004; citado en Mendoza, 2015: 83-118) señala que no hay conocimiento sin contexto, por lo que los habitantes deben explorar los entornos para comprender la realidad a la que se enfrentan. Las relaciones espaciales se sitúan por encima de las sociales aun cuando los espacios exteriores propician desinterés por sus características físico-espaciales y el estado actual del mobiliario y la vegetación.

Respecto a la participación, esta dimensión presenta los índices más bajos sin importar la escala espacial (conjunto habitacional o ciudad). Los habitantes mostraron un desinterés por involucrarse activamente en la comunidad. Un hecho que apoya este desinterés se asocia con la dispersión y la situación actual de los espacios comunes. En esta misma perspectiva son priorizados los espacios personales y las relaciones íntimas en el vínculo familiar.

Por último, en lo que respecta a las dimensiones de identidad y libertad, el deseo de permanencia en la ciudad se asocia moderadamente con el deseo de vivir en el conjunto habitacional. Espinoza (2010) relaciona el sentido de pertenencia con un indicador de satisfacción. Otro de los factores afectados refiere a la concepción de libertad, ya que de acuerdo con la ideología de Simmel, al tener una dimensión espacial menor, esto es la morfología física que presentan los conjuntos habitacionales, se restringe la libertad puesto que un contacto social más estrecho propicia una vigilancia más íntima (Lezama, 2002).

\section{Relaciones socio-espaciales para la estimación de habitabilidad en los conjuntos habitacionales}

La descripción de las características espaciales de los conjuntos habitacionales en cuanto a su funcionalidad, diseño, equipamiento, entre otros, favorece la identificación de las condiciones que propician los valores encontrados de habitabilidad. A continuación, se presentan los 
principales hallazgos en torno a la habitabilidad y relaciones socialesespaciales en los $\mathrm{CH}$.

Los Encinos Horizontal es un $\mathrm{CH}$ mayormente afectado en sus relaciones sociales. El contexto se percibe como un espacio de paso, donde el interés principal del residente es llegar al ámbito familiar. Se presenta un deseo inminente de permanencia en la ciudad, pero sin una búsqueda por relacionarse con otros en esta escala espacial ni por participar en ella. Dicho deseo de permanencia se asocia a la apertura espacial del $\mathrm{CH}$ al contar con mayores dimensiones físicas que los otros $\mathrm{CH}$, facilidad de desplazamiento, accesibilidad y pendiente pronunciada en toda la planta arquitectónica.

Por su parte, Los Encinos vertical se exhibe como un $\mathrm{CH}$ principalmente afectado por el nivel de inseguridad. Esta situación se propicia por problemas de alumbrado público, lotes baldíos, basura y poca vigilancia policiaca, por mencionar algunos. Sus residentes tienen bajo perfil socioeconómico y cultural. Las relaciones identitarias a nivel ciudad se mantienen en la cima no obstante que las conexiones viales son deficientes. La evaluación de los niveles de libertad en cuanto a apertura espacial se encuentra como un aspecto positivo ya que, si bien su organización espacial es vertical, el posicionamiento del $\mathrm{CH}$ y la pendiente del terreno favorecen que el espacio adquiera la cualidad de mirador a sus alrededores, lo que sugiere un acercamiento a la escala de ciudad.

En el caso de Villas del Rey I, este $\mathrm{CH}$ es visto como un espacio donde la relación ser/espacio es fundamental en una escala espacial menor a la de ciudad. Sus residentes se interesan por los espacios comunes internos, mismos que mantienen para contar con un espacio de descanso/ ocio. La menor cantidad de viviendas y la ubicación del $\mathrm{CH}$ permite que los problemas de congestionamiento vial sean menores. De manera similar a Encinos horizontal, se presenta un desinterés por involucrarse socialmente en la ciudad, ya sea a nivel de establecer cualquier tipo de relación o de participar en eventos voluntarios para su mejoramiento.

Por último, Punta Banda es un $\mathrm{CH}$ con una ubicación estrategia en la ciudad. Posee una comunicación estratégica en una zona altamente urbanizada y con facilidad de acceso al transporte público. El deseo de permanencia en la ciudad se acompaña del sentido de libertad en los lugares que se habitan y por la facilidad de llegada a la zona costera del puerto o a puntos de interés en la ciudad. Se identifica una relación prioritaria entre el ser y el espacio, vinculada a las relaciones identitarias con la ciudad, por la cercanía a áreas de esparcimiento y culturales en el entorno inmediato. Sin embargo, se tiene un patrón de baja o nula participación en la ciudad.

\section{CONCLUSIONES Y RECOMENDACIONES}

El proceso de habitar involucra relaciones sociales que acontecen en el espacio público urbano lo que conlleva a una transformación espacial desde lo físico, social y cultural; con la intención de subsanar los 
requerimientos individuales y colectivos de los habitantes. Se destaca que el nivel de habitabilidad en los cuatro conjuntos habitacionales de estudio depende de mediaciones entre lo privado, lo común y lo público, donde las relaciones se establecieron de forma bidireccional entre lo que se vive, lo que se percibe y lo que es impuesto. Es conveniente precisar que estos hallazgos dan cuenta de las características específicas de la zona de estudio. Se recomienda ampliar la investigación a otras zonas para disponer de una mejor perspectiva en otras áreas geográficas del país.

De las características que llaman la atención en los cuatro conjuntos habitacionales es la conformación morfológica y funcional que presentan estos espacios, ya que los sitúa como una realidad ajena a su entorno circundante, donde los factores relacionados con la subsistencia se concentran en priorizar el bienestar personal, con un limitado interés en el exterior o el colectivo mismo. Este hecho influyó en los valores mínimos logrados en la dimensión de participación, ya reportada en la figura 3. Se coincide con lo señalado por Delgado (1999) al mencionar que, en este tipo de espacios, las relaciones sociales se caracterizan por ser apresuradas, impersonales y de conveniencia.

El habitante prefiere aislarse en su espacio interior.

\section{Estas acciones son un reflejo de \\ LA PROBLEMÁTICA SOCIAL}

que aqueja a los residentes

DE CONDOMINIOS DE INTERÉS SOCIAL DE ESTE ESTUDIO.

Las dimensiones con evaluación baja fueron: subsistencia, protección y participación, en los cuatro $\mathrm{CH}$ del estudio. Fue evidente la preocupación por la inseguridad, la baja participación en colectivo y en convivencias. El habitante prefiere aislarse en su espacio interior. Estas acciones son un reflejo de la problemática social que aqueja a los residentes de condominios de interés social de este estudio. En contraparte, se refiere un deseo de permanecer en la ciudad, hay un sentimiento de pertenencia.

A manera de cierre, es importante mencionar que la discusión del estudio sobre la habitabilidad y las relaciones socio-espaciales en los conjuntos habitacionales de interés social enriquece la visión y ofrece una nueva perspectiva al diseño del espacio habitable en Ensenada. No obstante, no se puede generalizar la relación entre el espacio exterior (urbano) y el interior (del conjunto habitacional y la vivienda), ya que los elementos del contexto son específicos de una realidad determinada y aun cuando se ha mencionado que las necesidades son las mismas en el tiempo y de cultura a cultura, tanto los satisfactores como el nivel e intensidad en la que se satisfacen van a depender del espacio y las circunstancias implicadas.

\section{Agradecimientos}

La realización de esta investigación fue posible gracias a la Universidad Autónoma de San Luis Potosí y a la Universidad Autónoma de Yucatán por medio de la Facultad de Ciencias del Hábitat y la 
Facultad de Arquitectura, respectivamente, y al Programa de Doctorado Interinstitucional en Ciencias del Hábitat, del que forma parte este trabajo.

\section{FUENTES DE CONSULTA}

Augé, M. (1998), Los no lugares, Editorial Gedisa, España.

Carrera, F. \& M. Balsells (2011), "Instrumento de Evaluación de Competencias Digitales para adolescentes en riesgo social”, EDUTEC-E Revista Electrónica de Tecnología Educativa, [En línea] http://edutec.rediris.es/ revelec2/revelec35, pp. 1-25, consultado el 14 de octubre de 2017.

Castells, M. (2004), La cuestión urbana, Siglo XXI Editores, México.

Delgado, M. (1999), El animal público, Anagrama, España.

Espinoza López, A. E., Gómez Azpeitia, G. (2010), "Hacia una concepción socio-física de la habitabilidad: espacialidad, sustentabilidad y sociedad". [En línea] http://www.redalyc.org/src/inicio/ArtPdfRed.jsp? $\mathrm{iCve}=94820714006$, consultado el 13 de mayo de 2016 .

Gazmuri Núñez, P. (2013), "Familia y habitabilidad en la vivienda: Aproximaciones metodológicas para su estudio desde una perspectiva sociológica", Arquitectura y Urbanismo, vol. XXXIV, no. 1, pp. 32-47.

Gehl, J. (2009), La humanización del espacio urbano, Editorial Reverté, España.

Giglia, Á. (2012), "El habitar y la cultura: perspectivas teóricas y de investigación", Anthropos-Universidad Autónoma Metropolitana, México.

Gottdiener, M. (1985), The Social Production of Urban Space, University of Texas, Texas.

Lefebvre, H. (2013), La producción del espacio, Capitán Swing, España.

Lezama, J. L. (2002), Teoría social, espacio y ciudad, Editorial El Colegio de México, Centro de Estudios Demográficos y de Desarrollo Urbano, México.

Mendoza García, J. (2015), "Otra mirada: la construcción social del conocimiento”, Polis, vol. 11, núm. 1, pp. 83-118.

Monnet, J. (1996), “Espacio público, comercio y urbanidad en Francia, México y Estados Unidos”, Alteridades, vol. 6, núm. 11, pp. 11-25.

Rémy, J., Voyé, L. (1974), La ville et L'urbanisation. Modalités d'analyse sociologique, Éditions J. Duculot, S.A. Belgique.

Sánchez-Corral, J. (2012), La vivienda "social" en México. Pasado, Presente y Futuro, JSa, México.

Saunders, P. (2011), Social Theory and the Urban Question, Richard T. LeGates and Frederic Stout, New York, pp. 13-51. 